\title{
Uncomplicated type A intramural hematoma: surgery or conservative approach? - conservative approach
}

\author{
Hitoshi Ogino \\ Department of Cardiovascular Surgery, Tokyo Medical University, Tokyo, Japan \\ Correspondence to: Hitoshi Ogino, MD, PhD. Department of Cardiovascular Surgery, Tokyo Medical University, 6-1-1 Nishishinjuku, Shinjuku-ku, \\ Tokyo 160-0023, Japan. Email: hogino@tokyo-med.ac.jp.
}

Submitted Apr 01, 2019. Accepted for publication Jun 20, 2019.

doi: 10.21037/acs.2019.06.10

View this article at: http://dx.doi.org/10.21037/acs.2019.06.10

In this article, "intramural hematoma (IMH)" is defined as the entity including both the original/pathological lesion of the aortic media without any primary entry (intimal tear) and the radio-/echo-graphical condition with the false lumen (FL) of aortic dissection (AD) completely thrombooccluded (excluding partially thrombosed) with or without a clearly-demonstrated intimal tear on imaging. The treatment strategies for IMH depend on the location of the $\mathrm{IMH}$, the degree/thickness of the IMH, and the presence of coexisting critical sequelae of acute type $\mathrm{A} A D$, such as pericardial effusion (PE)/cardiac tamponade, rupture, significant aortic regurgitation, and organ malperfusion. The patient's comorbidities and age also should be assessed when considering emergent/urgent open aortic repairs. The initial treatment strategies for type A IMH are discussed, focusing on the conventional approach for "uncomplicated" type A IMH.

The surgical indication for acute type A IMH remains controversial, because its natural history is more variable, compared with acute type B IMH. The ACCF/ AHA guideline in 2010 and the ESC guidance in 2014 recommend emergency open aortic repair, particularly for "complicated" type A IMH with the above-described critical sequelae of acute type $\mathrm{A} A \mathrm{AD}(1,2)$. However, surgical indications for "uncomplicated" type A IMH remain unclear with no indications described in either guideline. In the literature, mortality rates of medically-treated patients in the European and American series are higher (3-5), compared with the Asian series (6-8). The reasons for this are not clear, however, they might be due to differences in the medical care systems and medical treatments offered between the American/European and Asian countries. In
Japan, there are over 580 cardiovascular surgical centers in the country, including 42 hospitals in the small and crowded area of Tokyo where emergency medical and surgical treatments for acute $\mathrm{AD}$ are feasible.

For patients not requiring urgent surgical treatments, the conservative "wait-and-watch strategy" is appropriate, this includes optimal medical therapy with blood pressure and pain control and with appropriate serial imaging. Optimal medical treatment is directed at decreasing aortic wall stress by controlling heart rate and blood pressure as followed; intravenous beta blockade or calcium channel-blocking agents are given and titrated to a target heart rate of $60 \mathrm{bpm}$ or less. If the systolic blood pressures remains greater than $120 \mathrm{mmHg}$ after adequate heart rate control, angiotensinconverting enzyme inhibitors and/or other vasodilators are administered intravenously. With this conservative wait-and-watch strategy, the FL hematoma may resolve in some instances. However, in other patients the IMH may convert to a classical (double-barrel) $\mathrm{AD}$ or the aorta may dilate with thickening of the FL, in particular, during the initial 24-48 h (3-5). An ulcer-like-projection (ULP) cause by a small intimal tear sometimes appears in the thrombooccluded FL. Such a conversion or change of AD pattern would be associated with serious sequelae following acute type $\mathrm{A} \mathrm{AD.}$

Type A IMH tends to have a higher incidence of the above complications due to higher wall stress and tension on the ascending aorta compared with more stable type B IMH. The risk of conversion to classical type A AD despite optimal medical management is particularly high in patients with a dilated aorta to $48-50 \mathrm{~mm}$ or with an IMH thickness of $11 \mathrm{~mm}(6-8)$. In this settings, the initial 
treatment should be emergent or urgent surgery for most patients without extremely-high surgical risks. Interestingly, Uchida et al. demonstrated higher incidences of $\mathrm{PE} /$ cardiac tamponade in type A IMH compared with a classical type A $\mathrm{AD}$ (9). Pathology of aortic specimens collected at surgery revealed that $\mathrm{AD}$ tended to occur in the media closer to the adventitia. In other words, the actual incidence of "uncomplicated" type A IMH without PE might be lower. In cases with more than a moderate amount of $\mathrm{PE}$ and without the above surgical criteria of over $50 \mathrm{~mm}$ in aortic diameter and $11 \mathrm{~mm}$ of IMH thickness, pericardial drainage would be a useful treatment option particularly for high-risk or inoperable patients to improve the hemodynamics and preventing cardiac tamponade, thus avoiding more invasive open repairs (10).

In the conservative, wait-and-watch approach the interval of imaging evaluation is also important. However, there have been no clear recommendations for the appropriate interval guidelines. We believe that initial surveillance imaging should be carried out within 24 hours of the initial investigation, because $\mathrm{AD}$ is unstable during the super-acute phase of the first 24-48 h. Fortunately, the $\mathrm{IMH}$ thickness is easily recognized even in non-enhanced CT-scans. However, during this phase, conversion from IMH to IMH with a ULP or to classical AD can potentially occur. In cases with enlarged ascending aorta or thickened IMH, if possible, contrast enhanced CT-scan is recommended to demonstrate all features clearly at the second assessment.

In cases with 'uncomplicated' type A IMH with the maximum aortic diameter less than $50 \mathrm{~mm}$ and the IMH thickness less than $11 \mathrm{~mm}$, the first line treatment might be a wait-and-watch strategy as determined by a multidisciplinary aorta team including sufficient surgical support. However, during conservative treatment, the occurrence of critical sequelae such as $\mathrm{PE} /$ cardiac tamponade, rupture, significant aortic regurgitation, and organ malperfusion, or further dilatation of the aorta over $50 \mathrm{~mm}$ and/or extension of IMH over $11 \mathrm{~mm}$, change of treatment strategy to urgent open aortic repair is recommended.

\section{Acknowledgments}

None.

\section{Footnote}

Conflicts of Interest: The author has no conflicts of interest to declare.

\section{References}

1. Hiratzka LF, Bakris GL, Beckman JA, et al. 2010 ACCF/AHA/AATS/ACR/ASA/SCA/SCAI/SIR/STS/ SVM guidelines for the diagnosis and management of patients with Thoracic Aortic Disease: a report of the American College of Cardiology Foundation/American Heart Association Task Force on Practice Guidelines, American Association for Thoracic Surgery, American College of Radiology, American Stroke Association, Society of Cardiovascular Anesthesiologists, Society for Cardiovascular Angiography and Interventions, Society of Interventional Radiology, Society of Thoracic Surgeons, and Society for Vascular Medicine. Circulation 2010;121:e266-369.

2. Erbel R, Aboyans V, Boileau C, et al. 2014 ESC Guidelines on the diagnosis and treatment of aortic diseases: Document covering acute and chronic aortic diseases of the thoracic and abdominal aorta of the adult. The Task Force for the Diagnosis and Treatment of Aortic Diseases of the European Society of Cardiology (ESC). Eur Heart J 2014;35:2873-926.

3. Evangelista A, Mukherjee D, Mehta RH, et al. Acute intramural hematoma of the aorta: a mystery in evolution. Circulation 2005;111:1063-70.

4. Estrera A, Miller C 3rd, Lee TY, et al. Acute type A intramural hematoma: analysis of current management strategy. Circulation 2009;120:S287-91.

5. Harris KM, Braverman AC, Eagle KA, et al. Acute aortic intramural hematoma: an analysis from the International Registry of Acute Aortic Dissection. Circulation 2012;126:S91-6.

6. Kaji S, Nishigami K, Akasaka T, et al. Prediction of progression or regression of type A aortic intramural hematoma by computed tomography. Circulation 1999;100:II281-6.

7. Kitai T, Kaji S, Yamamuro A, et al. Clinical outcomes of medical therapy and timely operation in initially diagnosed type a aortic intramural hematoma: a 20-year experience. Circulation 2009;120:S292-8. 
8. Song JK, Yim JH, Ahn JM, et al. Outcomes of patients with acute type a aortic intramural hematoma. Circulation 2009;120:2046-52.

9. Uchida K, Imoto K, Takahashi M, et al. Pathologic characteristics and surgical indications of superacute type A intramural hematoma. Ann Thorac Surg

Cite this article as: Ogino $\mathrm{H}$. Uncomplicated type A intramural hematoma: surgery or conservative approach? conservative approach. Ann Cardiothorac Surg 2019;8(5):558-560. doi: $10.21037 /$ acs.2019.06.10
2005;79:1518-21.

10. Hayashi T, Tsukube T, Yamashita T, et al. Impact of controlled pericardial drainage on critical cardiac tamponade with acute type A aortic dissection. Circulation 2012;126:S97-101. 